\title{
Capacity of State and Territorial Health Agencies To Prevent Foodborne Illness
}

\author{
Richard E. Hoffman, ${ }^{*}$ Jesse Greenblatt, $†$ Bela T. Matyas, $\ddagger$ Donald J. Sharp,§ Emilio Esteban, $\uparrow$ \\ Knachelle Hodge, ${ }^{*}$ and Arthur Liang§
}

The capacity of state and territorial health departments to investigate foodborne diseases was assessed by the Council of State and Territorial Epidemiologists from 2001 to 2002 with a self-administered, Web-based survey. Fortyeight health departments responded (47 states and 1 territory). The primary reason for not conducting more active case surveillance of enteric disease is lack of staff, while the primary reasons for not investigating foodborne disease outbreaks are limited staff and delayed notification of the outbreak. Sixty-four percent of respondents have the capacity to conduct analytic epidemiologic investigations. States receiving Emerging Infections Program (EIP) funding from the Centers for Disease Control and Prevention more often reported having a dedicated foodborne disease epidemiologist and the capability to perform analytic studies than non-EIP states. We conclude that by addressing shortages in the number of dedicated personnel and reducing delays in reporting, the capacity of state health departments to respond to foodborne disease can be improved.

$\mathrm{F}$ oodborne illnesses are common. Each year an estimated 76 million foodborne illnesses occur, with 325,000 hospitalizations and 5,000 deaths (1), and a recent estimate of annual costs for medical treatment, productivity loss, and premature deaths resulting from these illnesses is \$6.5 billion (2). The National Food Safety Initiative (NFSI) was started in 1997 as an effort to decrease the incidence and risk for foodborne illness (3). The NFSI ended in 2001, but at the Centers for Disease Control and Prevention (CDC),

${ }^{*}$ Council of State and Territorial Epidemiologists, Atlanta, Georgia, USA; †New Hampshire Department of Health and Human Services, Concord, New Hampshire, USA; ‡Massachusetts Department of Public Health, Boston, Massachusetts, USA; $\S$ Centers for Disease Control and Prevention, Atlanta, Georgia, USA; and TU.S. Department of Agriculture, Alameda, California, USA the former NFSI funding and activities have been institutionalized as an ongoing food safety program. Continued progress on the part of regulators and industry to improve food safety are dependent on local, state, and federal agencies' ability to conduct epidemiologic and laboratory investigations that identify the offending agents and link them with specific foods.

Improvements in detecting and investigating foodborne illnesses were made during the 1990s when CDC implemented the Foodborne Diseases Active Surveillance Network (FoodNet), a component of the Emerging Infections Programs (EIP), and PulseNet (4,5). EIP is a network of epidemiology programs in state health departments that is funded and coordinated by CDC. It is intended to be a national resource for surveillance and epidemiologic research that goes beyond the routine public health department functions. Active, laboratory-based surveillance is the foundation of 2 core EIP projects conducted at all sites: Active Bacterial Core Surveillance and Foodborne Disease Active Surveillance. Ten states currently receive EIP support from CDC. PulseNet, unlike EIP, is intended to be a national molecular subtyping network for foodborne disease surveillance. It was established by the CDC in 1996 to facilitate subtyping bacterial foodborne pathogens by state health department laboratories. Even after implementing FoodNet and PulseNet, much work remains to improve the state and local public health agencies' capacity to detect and investigate foodborne disease.

In 1999, CDC provided funding to both Council of State and Territorial Epidemiologists (CSTE) and the Association of Public Health Laboratories (APHL) to conduct assessments of states' foodborne disease investigation capacity. The purpose of both assessments was to determine priorities for improving food safety program support. The CSTE assessment was intended to concentrate 
primarily on state and territory health departments' capacity to monitor and investigate foodborne illness. This report presents the results of the CSTE survey, which was conducted from October 2001 to March 2002, of 48 state and territorial health agencies,

An expert CSTE committee, composed of state and local epidemiologists from Colorado; Philadelphia; and Los Angeles County, California; an environmentalist from DeKalb County, Georgia; a state laboratorian from Rhode Island; staff from the CDC's National Center for Infectious Disease, Division of Bacterial and Mycotic Diseases-Food Safety Office; and CSTE staff from its national office developed a survey instrument that was pilot-tested in 6 states and subsequently revised. The final instrument consisted of 106 questions. We present analyses of selected questions; a complete tabulation of all results and display of the questionnaire are available from the CSTE website (6). The data can be used as a baseline reference for future surveys of state and territorial capacity to investigate foodborne disease.

\section{Methods}

The assessment instrument was a self-administered, Web-based survey. Respondents were state and territorial epidemiologists with knowledge in the area of foodborne diseases. The assessment was conducted from October 2001 through February 2002, and during the 5-month survey period, reminder telephone calls and emails were made from the CSTE national office to health agencies that had not yet responded.

The instrument's 106 questions covered background information about the responding agency, epidemiologic surveillance capacity to identify sporadic and outbreakrelated illnesses; capacity to investigate and respond to outbreaks; public health infrastructure to support food safety activities, defined as staffing, facilities, equipment, supplies, information, communication between epidemiology and laboratory units, and education and training of staff; and legal authority of the agency. We restricted results in this article to questions pertaining to agency capacity and operations, barriers to the investigation of foodborne diseases, and staffing of the epidemiology program (a subset of "barriers").

Forty-eight health departments responded (47 states [response rate $=94 \%$ ] and 1 territory [Guam]); Pennsylvania, Illinois, Nevada, and Puerto Rico did not submit responses. Some questions did not elicit 48 responses. Responses reflect the perspective of the epidemiology program in the agency. The frequency and percentage for each response were calculated on the basis of the total number of responses to that question. Percentages are rounded to the nearest integer. The phrasing of questions in tables in the Results section has, in some instances, been shortened from the exact words used in the questionnaire.

We also examined responses by whether the responding agency received EIP funding from CDC (8 of 9 EIP sites that were funded at the time responded to the survey: Colorado, Connecticut, Georgia, New York, Minnesota, Oregon, Tennessee, and California/San Francisco Bay) and whether the responding agency was a jurisdiction with large population (10 largest population states in 2000 U.S. census; number of respondents $=8$; population range 33,871,648-8,186,453), medium population (states ranked 11th to 20th in population in 2000 census; number of respondents $=10$; population range $8,049,313$ $5,130,632$ ), or small population (the remaining states and 1 territory; number of respondents $=30$; population range 4,919,479-154,805). The term "small population states" includes 29 states and 1 territory (Guam). The 8 responding EIP sites included 3 large, 1 medium, and 4 small population states.

\section{Results}

Forty percent of the states receive laboratory reports electronically. The primary reason reported for not conducting more active case surveillance is lack of staff. The primary reasons reported for not investigating foodborne disease outbreaks are limited staff and delayed reporting of the outbreak. Sixty-four percent of respondents have the capacity to conduct analytic epidemiologic investigations. Thirty-five percent of respondents have a protocol to guarantee chain of custody for food specimens. Eighty-one percent of respondents can obtain public health laboratory, environmental health, and sanitation support 24 hours per day. Fifty-four percent of respondents have broadcast fax or email capability to hospital emergency rooms and to physicians (Tables 1-3).

We did not find that EIP sites always reported more capacity and more advanced operations than non-EIP sites. A greater percentage of EIP sites than non-EIP sites reported adequate capacity to conduct analytic epidemiologic studies (88\% vs. 59\%) and having a regulation or statute specifically requiring the submission of certain enteric isolates to the public health laboratory ( $75 \%$ vs. $50 \%$ ). On the other hand, a smaller percentage of EIP sites than non-EIP sites reported having the capacity to broadcast faxes to hospital emergency departments (50\% vs. $55 \%$ ) and to conduct syndromic surveillance for diarrheal disease $(0 \%$ vs. $18 \%$ ). The percentage of EIP sites having a protocol to guarantee chain of custody for food environmental specimens was nearly the same as for non-EIP sites (38\% vs. $36 \%)$.

Likewise, we found that large population states did not consistently have more capacity and more advanced operations than medium or small population states or 
Table 1. Capacity and operations of epidemiologic, laboratory, and environmental programs in state and territorial health departments

\begin{tabular}{|c|c|c|c|c|}
\hline Question & $\mathrm{n}$ & $\%$ yes & $\%$ no & $\%$ not sure \\
\hline $\begin{array}{l}\text { Does the Epidemiology Office have the ability to receive electronic laboratory } \\
\text { reporting of enteric diseases? }\end{array}$ & 48 & 40 & 60 & \\
\hline Do you routinely collect stool samples for testing? & 47 & 98 & 2 & \\
\hline Do you routinely collect vomitus for testing? & 47 & 38 & 62 & \\
\hline Do you have broadcast fax or email capability to: (list all that apply) & 48 & & & \\
\hline Other health departments within the state & & 88 & & \\
\hline Hospital infection control specialists & & 77 & & \\
\hline Hospital emergency rooms & & 54 & & \\
\hline Physicians & & 50 & & \\
\hline Other state health departments & & 40 & & \\
\hline Other & & 19 & & \\
\hline Do you conduct syndromic surveillance for diarrheal disease? & 48 & 15 & 77 & 8 \\
\hline $\begin{array}{l}\text { Do you have adequate capacity to conduct an analytic epidemiologic investigation, } \\
\text { i.e., case-control or cohort studies? }\end{array}$ & 47 & 64 & 30 & 6 \\
\hline \multicolumn{5}{|l|}{ Does your agency have legal authority to: } \\
\hline Collect reports of suspected enteric diseases? & 48 & 90 & 4 & 6 \\
\hline Perform on-the-spot emergency environmental/sanitation inspections? & 48 & 85 & 4 & 10 \\
\hline Exclude sick or infected employees from food handling duties? & 48 & 83 & 13 & 4 \\
\hline $\begin{array}{l}\text { Share information related to foodborne outbreaks with federal agencies, e.g., } \\
\text { USDA, FDA, and CDC?*}\end{array}$ & 47 & 83 & 4 & 13 \\
\hline Close a food service facility? & 48 & 81 & 15 & 4 \\
\hline Collect reports of clinical syndromes? & 48 & 71 & 19 & 10 \\
\hline Embargo or condemn food? & 47 & 66 & 11 & 23 \\
\hline $\begin{array}{l}\text { Is there a regulation/statute specifically requiring submission of certain enteric } \\
\text { isolates to the public health laboratory? }\end{array}$ & 48 & 54 & 38 & 8 \\
\hline $\begin{array}{l}\text { Does the department of health have a protocol to guarantee chain of custody for } \\
\text { food environmental specimens? }\end{array}$ & 48 & 35 & 48 & 17 \\
\hline
\end{tabular}

territories. Seventy-five percent of large states, $90 \%$ of medium states, and $52 \%$ of small population states reported adequate capacity to perform analytic epidemiologic studies. Thirty-eight percent of large states, $30 \%$ of medium, and $67 \%$ of small states reported the capacity to broadcast fax to hospital emergency departments. The differences between state size and having a chain of custody protocol for food specimens were relatively small (50\% of large, $40 \%$ of medium, and $30 \%$ of small population states), while the differences in percentage reporting a legal requirement to submit certain enteric isolates to the public health laboratory were relatively large: $38 \%$ of large states, $70 \%$ of medium states, and $53 \%$ of small states.

As for factors that limit ability to investigate outbreaks, the most common reason given by both EIP and non-EIP sites was "delayed notification" (88\% vs. 83\%). The percentage of EIP sites and non-EIP sites reporting "limited staff" (63\% vs. $68 \%)$ and "lack of importance" (50\% vs. $45 \%)$ were similar. Delayed notification was the most frequent reason given by large (75\%), medium (100\%), and small (80\%) population states for not investigating outbreaks. Seventy percent of small states compared to $70 \%$ of medium states and $50 \%$ of large states reported limited staff as a reason for not investigating outbreaks.

Seventy-two percent of EIP sites versus $83 \%$ of nonEIP sites reported having laboratory support 24 hours per day, whereas $75 \%$ of EIP sites compared to $43 \%$ of nonEIP sites reported having a dedicated enteric/foodborne epidemiologist. For these same two questions, $73 \%$ of small population states versus $100 \%$ of large and $90 \%$ of medium states had laboratory support 24 hours per day, and $75 \%$ of large and $80 \%$ of medium states had a dedicated enteric/foodborne disease epidemiologist compared to $30 \%$ of small population states. Lastly, during outbreaks, $100 \%$ of EIP sites versus $68 \%$ of non-EIP sites reported that they had enough people to enter data. For this question, the differences between large, medium, and small population states were relatively small $(88 \%, 70 \%$, $70 \%$, respectively).

\section{Discussion and Conclusion}

In the United States, the primary responsibility for foodborne disease surveillance and investigation lies with state, territorial, and local health agencies, with technical backup and funding support from CDC and other federal agencies, including the Food and Drug Administration and the Food Safety and Inspection Service of the U.S. Department of Agriculture. Within a state public health agency, reducing the incidence of foodborne disease requires a sensitive surveillance system, timely epidemiologic investigation of sporadic cases and outbreaks with the most current laboratory technologies, and coordina- 
Table 2. Barriers to investigating foodborne disease in state and territorial health departments

\begin{tabular}{|c|c|c|c|c|}
\hline Question & $\mathrm{n}$ & $\%$ yes & $\%$ no & $\%$ not sure \\
\hline $\begin{array}{l}\text { Of the outbreaks that are not investigated, which factors most limit your } \\
\text { ability to investigate? (list all that apply) }\end{array}$ & 48 & & & \\
\hline Delayed notification & & 83 & & \\
\hline Limited staff & & 67 & & \\
\hline Lack of apparent importance & & 46 & & \\
\hline Laboratory capacity & & 21 & & \\
\hline Jurisdictional issues & & 19 & & \\
\hline Political consideration & & 13 & & \\
\hline Expertise & & 13 & & \\
\hline Other & & 13 & & \\
\hline Travel policy constraints & & 11 & & \\
\hline Statistical support & & 8 & & \\
\hline Ability to pay overtime & & 8 & & \\
\hline $\begin{array}{l}\text { In outbreaks in which food specimens were not submitted, what } \\
\text { were the barriers to laboratory testing? }\end{array}$ & 47 & & & \\
\hline Leftovers not available & & 98 & & \\
\hline Wrong food collected & & 32 & & \\
\hline Unnecessary & & 17 & & \\
\hline Other & & 13 & & \\
\hline No capability for food testing, i.e., laboratory equipment & & 11 & & \\
\hline Insufficient expertise at laboratory & & 6 & & \\
\hline Too expensive & & 4 & & \\
\hline Do you feel there are barriers for conducting more active case surveillance? & 48 & 88 & 8 & 4 \\
\hline If yes, which of the following reasons apply: (list all that apply) & 42 & & & \\
\hline Lack of staff & & 81 & & \\
\hline Too time-consuming & & 60 & & \\
\hline Other & & 33 & & \\
\hline Low priority & & 29 & & \\
\hline Lack of expertise & & 12 & & \\
\hline
\end{tabular}

tion of epidemiology, environmental, and laboratory programs.

This report is the fourth in a series by CSTE to assess epidemiologic capacity in state and territorial health departments. The 3 previously published surveys concerned overall capacity, maternal and child health capacity, and chronic disease capacity (7-10). While infectious disease capacity was addressed in the overall survey conducted from November 2001 through April 2002, this report is the most detailed analysis of states' and territories' foodborne disease capacity to date. The findings in the overall capacity report concerning reasons why outbreaks were not investigated by the state health department are similar to findings in our report: of 42 respondents 40 (95\%) reported delayed notification of case reports, 33 (79\%) reported limited staff, and 31 (74\%) reported competing priorities for use of public health resources (7).

In the areas of foodborne disease surveillance and investigation, our report documents that the aggregate perception of a large sample of epidemiologic leaders in state and territorial health departments is that, as of 2002, more resources were needed. The data are self-reported and do not include responses from a few large states and Puerto Rico. The survey found that lack of staff was the most frequent reason (81\% of respondents) for not conducting more active case surveillance, and the most frequent reasons given for not investigating outbreaks were delayed notification (83\%) and limited staff (63\%) (Table 2).

Our findings are also consistent with a 50-state survey conducted by the General Accounting Office in 2000 to 2001 (2). That survey found, for example, 32 (64\%) of 50 states indicated that more trained epidemiologists were needed at the state level to investigate outbreaks, and 44 (88\%) of 50 states indicated that more trained epidemiologists were needed at the local level to investigate outbreaks.

If a state or territory had more epidemiologists to conduct surveillance, fewer delays would likely occur in recognition of outbreaks, and more expertise would be available to conduct investigations. Thus, by addressing shortages in the number of dedicated personnel and reducing delays in reporting, the capacity of state health departments to respond to foodborne disease can be improved.

We also performed comparisons of EIP to non-EIP sites and of large, medium, and small population states. Only 8 of 10 possible large population states and 8 of 9 EIP states were included, so the analyses must be interpreted cautiously. Because these comparisons were conceived after the survey data had been collected, we did not perform analytic statistical tests, which could be misinterpreted. 
Table 3. Staffing in epidemiology programs for foodborne disease surveillance and investigation

\begin{tabular}{|c|c|c|c|c|}
\hline Question & $\mathrm{n}$ & $\%$ yes & $\%$ no & $\%$ not sure \\
\hline For sporadic cases, do you have enough people to: & 48 & & & \\
\hline Compare to standardized case definition & & 85 & 15 & \\
\hline Enter data & & 79 & 19 & 2 \\
\hline Review data for consistency and completeness & & 71 & 27 & 2 \\
\hline During outbreaks, do you have enough people to: & 48 & & & \\
\hline Compare to standardized case definition & & 90 & 10 & \\
\hline Enter data & & 73 & 19 & 8 \\
\hline Review data for completeness and consistency & & 71 & 23 & 6 \\
\hline $\begin{array}{l}\text { In your enteric/foodborne disease epidemiology program, do you have sufficient } \\
\text { statistical support? }\end{array}$ & 47 & 45 & 47 & 9 \\
\hline Do you have a dedicated enteric/foodborne disease epidemiologist at your agency? & 48 & 48 & 50 & 2 \\
\hline If yes to question above, what is the highest level of education of the epidemiologist? & 23 & & & \\
\hline Masters degree & & 61 & & \\
\hline Doctoral degree & & 26 & & \\
\hline Bachelor degree & & 13 & & \\
\hline $\begin{array}{l}\text { During an outbreak investigation, do epidemiologists routinely accompany } \\
\text { environmental health/sanitation specialist(s)? }\end{array}$ & 48 & 44 & 50 & 6 \\
\hline Is there a 24-hour on-call response mechanism for foodborne disease issues? & 48 & 96 & 4 & \\
\hline Can you get public health laboratory support $24 / 7 / 365 ?$ & 48 & 81 & 15 & 4 \\
\hline Can you get environmental health/sanitation support 24 hours per day? & 48 & 60 & 23 & 17 \\
\hline Do your epidemiologists receive training in environmental food facility inspections? & 48 & 13 & 85 & 2 \\
\hline Do your environmental health/sanitation specialists receive training in epidemiology? & 48 & 63 & 33 & 4 \\
\hline
\end{tabular}

Our descriptive findings are presented for interest and generation of hypotheses. We observed that EIP sites more frequently stated they had a dedicated foodborne disease epidemiologist, the capacity to perform analytic epidemiologic studies, and sufficient personnel to enter data during an outbreak than non-EIP sites. These findings would be expected, however, because 1 of the 2 core EIP projects is FoodNet. In other measures of capacity and program structure not specifically funded by the EIP programs, such as on-call laboratory support, not much difference existed between EIP and non-EIP sites.

The findings of this report do not indicate the quantity of resources needed to ensure sufficient capacity to protect the nation, and the survey results do not direct the allocation of new resources. One approach to this issue would be to assess the reported incidence of enteric disease and foodborne outbreaks with respect to self-reported capacity to monitor and investigate foodborne disease. However, the nation's system for identifying, investigating, and reporting foodborne diseases has not produced consistent and reliable data of adequate quality to perform such analyses. For example, in 1997, a total of 27 states and 3 territories reported zero outbreaks (10). More outbreaks must have occurred than were reported. Whatever the various reasons for such underreporting, the existing surveillance data are insufficient for addressing programmatic issues, such as where to invest in the public health system and what improvements in public health may reasonably be expected from such investment. Nevertheless, analyses are not needed to justify that every state and territory needs 24 hours per day epidemiologic, laboratory, and environ- mental health and sanitation on-call response capacity, as well as the capacity to communicate with public health and medical care providers, policymakers, and the public.

The analyses in this report provide a picture of the status of the nation at a time just before the distribution in 2002 of more than $\$ 1$ billion to state, territorial, and local health agencies to improve bioterrorism response and preparedness capacity. Several criteria exist for the mitigation of foodborne illness listed in the bioterrorism preparedness cooperative agreement award notice and grant guidelines (Procurement and Grants Office, CDC, Announcement No. 99051). For example, having a formal outbreak investigation team is an illustration of focus area A (preparedness planning and readiness) of the bioterrorism preparedness cooperative agreement criteria; $70 \%$ of the respondents reported having this capacity. One of the guidelines in focus area $G$ (education and training) is financial support by the state health agency for enteric disease and foodborne illness continuing education; more than half of the respondents in this survey reported that their agency provides this financial support. Although only $54 \%$ of states and territories reported that they could send broadcast faxes of health information to emergency departments, this particular capacity is a high priority for bioterrorism preparedness and is almost certain to have been further improved since the survey was completed.

In addition to the food safety minimum performance and capacity standards for epidemiology and surveillance adopted by CSTE as a position statement in 2003 (11), we recommend that for the short-term, objective measures of foodborne disease surveillance, reporting, and investigation 
be developed by local, state, and federal agencies. For example, the intervals from enteric disease onset until the case is reported to CDC may be measured in each state agency. Such measures can be used to indicate areas of need, to document areas of improvement, and to support the appropriation of new funds and the allocation of resources in lieu of enteric disease incidence.

This survey and the surveys of overall, maternal and child health, and chronic disease epidemiologic capacity demonstrate a need for a larger workforce of epidemiologists. In response to the surveys, CSTE convened a workforce summit of leaders from within the CSTE organization, CDC, the Association of State and Territorial Health Officers, the American Public Health Association, and the Association of Schools of Public Health in January 2004 (12). In addition, at its annual meeting held in June 2004, the CSTE membership approved a resolution calling for an annual National Epidemiologist Awareness Day to bring attention to the work of epidemiologists in protecting the nation's health (12). While this report and the mentioned activities of CSTE are specific to disease prevention by states and territories in the United States, similar capacities may be needed by public health agencies in other regions of the world, such as the European Union and the WHO Global Salm-Surv programme. We hope that the survey design and the results will provide guidance and comparisons for readers in other countries.

\section{Acknowledgments}

The authors thank Latoya Osmani for her work in data collection and management and initial analyses and Patrick McConnon and Lakesha Robinson for providing valuable support and critique during the development of this manuscript.

CSTE conducted this project with support from its cooperative agreement U60/CCU007277-10 with the CDC's National Center for Infectious Disease/Division of Bacterial and Mycotic Diseases-Food Safety Office.

Dr. Hoffman is adjunct associate professor in the department of Preventive Medicine and Biometrics, University of Colorado Health Sciences Center, Denver, Colorado. He provides private consultation as a medical epidemiologist.

\section{References}

1. Mead PS, Slutsker L, Dietz V, McCaig LF, Bresee JS, Shapiro C, et al. Food-related illness and death in the United States. Emerg Infect Dis. 1999;5:607-25.

2. United States Government Accounting Office. Report \#01-973 on Food Safety Surveillance Systems, September 2001. Available from http://www.gao.gov/new.items/d01973.pdf

3. Food safety from farm to table: a national food safety initiativereport to the President May 1997. Available from http://www.foodsafety.gov/ fsg/vltfarm.html

4. Pinner RW, Rebmann CA, Schuchat A, Hughes JM. Disease surveillance and the academic, clinical, and public health communities. Emerg Infect Dis. 2003;9:781-7.

5. Swaminatha B, Barrett TJ, Hunter SB, Tauxe RV, the CDC PulseNet Task Force. PulseNet: the molecular subtyping network for foodborne bacterial disease surveillance, United States. Emerg Infect Dis. 2001;7:382-9.

6. Council of State and Territorial Epidemiologists. National assessment of epidemiologic capacity in food safety programs: findings and recommendations. September 2002. Available from http://www.cste.org/ pdffiles/fsreportfinal.pdf

7. Council of State and Territorial Epidemiologists. National assessment of epidemiologic capacity in public health: findings and recommendations. March 2003. Available from http://www.cste.org/pdffiles/ ecacover1.pdf

8. Council of State and Territorial Epidemiologists. National assessment of epidemiologic capacity in maternal and child health: findings and recommendations. December 2002. Available from http://www. cste.org/pdffiles/mchfinal.pdf

9. Centers for Disease Control and Prevention. Surveillance for foodborne disease outbreaks—United States, 1993-1997. MMWR Morb Mortal Wkly Rep. 2000;49(SS-1):10.

10. Council of State and Territorial Epidemiologists. National assessment of epidemiologic capacity in chronic disease: findings and recommendations. September 2004. Available from http://www.cste.org/ pdffiles/New\%20Features/CSTEChronicReportFINAL.pdf

11. Council of State and Territorial Epidemiologists. Position statements 2003: state food safety minimum performance/capacity standards for epidemiology and surveillance. Available from http://www.cste.org/ PS/2003pdfs/2003finalpdf/03-ID-02Revised.pdf

12. Council of State and Territorial Epidemiologists. Position statements 2004: national epidemiologist awareness day. Available from http://www.cste.org/ps/2004pdf/04-EC-03-final.pdf

Address for correspondence: Richard E. Hoffman, 8155 Fairmount Dr, Unit 511, Denver, CO 80230, USA; fax: 303-343-3054; email: rehoffman49@msn.com

Use of trade names is for identification only and does not imply endorsement by the Public Health Service or by the U.S. Department of Health and Human Services.

\section{emerging infectious diseases $\mathrm{O} / \mathrm{l}$ ine}

\section{www.cdc.gov/eid}

To receive tables of contents of new issues send an email to listserve@cdc.gov with subscribe eid-toc in the body of your message. 